\title{
Two Ways to Look at the Transition Process of the NICE Guidelines on the Management of AAA from the Proposed to the Final Version
}

\author{
Raman Uberoi $^{1} \cdot$ Michael Jenkins $^{2}$
}

Published online: 27 May 2020

(C) Springer Science+Business Media, LLC, part of Springer Nature and the Cardiovascular and Interventional Radiological Society of Europe (CIRSE) 2020

\section{From: Raman Uberoi}

Since we published the Editorial [1] regarding the proposed NICE (UK National Institute for Health and Care Excellence) guidelines on the management of abdominal aortic aneurysm (AAA), the final guidelines have now been published [2]. It is clear that this has been one of the most difficult guidance documents ever produced by NICE, as demonstrated not least by the amount of time it has taken to finally release it.

Much of the guidance is unchanged, but crucially NICE guidelines have done a complete about-turn on the key contentious recommendations in section 1.5, to now offer endovascular aortic repair (EVAR) to patients with complex copathology and to those that are anesthetically unfit for open repair. However, they have maintained the guidance for only doing complex EVAR under strict protocols for research. The direction of travel, however, remains that more patients should undergo open surgery wherever possible. This dramatic change will be welcomed by the majority of stakeholders, and NICE should be congratulated on having taken a more sensible and pragmatic approach, and having listened to the extensive feedback they received. Many of us felt the rigid ban on EVAR in the original guidance was perhaps a bit overzealous and a more measured approach to EVAR is what the majority of operators were calling for. The guidelines now allow clinicians to advise and support patients to deliver the most appropriate treatment, which for many will be open surgery as the most durable treatment for those fit enough to benefit from it, but also allow endovascular intervention in cases where surgery is not appropriate. The ability to continue with both standard and custom EVAR is also crucial in furthering research and development into better endovascular devices for the future.

However, this dramatic about-turn does raise questions as well to the workings of NICE. How was the decision to modify these guidelines made? The purpose of the consultation process undertaken by NICE, with stakeholders following a draft proposal, is to ensure that no important piece of evidence is overlooked. They have not, however, presented any new

Raman Uberoi and Michael Jenkins contributed equally to the article and should be both first authors.

Raman Uberoi

Raman.Uberoi@ouh.nhs.uk

Michael Jenkins

michael.jenkins5@nhs.net

1 Radiology Department, John Radcliff Hospital, Oxford, UK

2 Imperial College Healthcare NHS Trust, St Mary's Hospital, London, UK 
major literature evidence to fundamentally change guidelines 1.5 on the use of EVAR. On the contrary, the additional observational studies reviewed showed similar trends for outcomes for both open surgical repair and EVAR as to randomized trials, in particular the higher postoperative mortality for EVAR of $24 \%$. What is more, there has also been the unprecedented publication of a separate letter from the NICE aneurysm guidance development committee (GDC) who reviewed the evidence and distanced themselves from the revised NICE guidelines 1.5 [3]. This is very unfortunate, and it sadly gives the impression that despite the evidence weighed up by the NICE guidance committee, this was overruled by the NICE board who may or may not have reviewed the same data. Was this purely in response to external pressure rather than the weight of new evidence given to the committee? We can only speculate here. Under the rules, changes have to be agreed by the GDC.

There is a lot to commend in the current version of the NICE guidelines in improving patient care in the management of AAA, and we must not lose sight of this. NICE has a fantastic national and international reputation for developing guidance, but the whole document risks being tarnished by the clear disagreement by the committee appointed to independently review the evidence and the NICE board, potentially damaging NICE's reputation in the long term.

As we have stated previously, there needs to be a sensible balanced approach to abdominal aneurysm repair, with decisions made on the basis of what is in the best interests of patients and founded on good evidence. This does not mean treating all patients, but triaging those that would do best in the medium to long term and not just 30 days after surgery. This, in practice, means open surgery for the majority of younger fitter patients, and EVAR for those with complex copathology or higher anesthetic risk, but not those with short life expectancies. This is best done in the setting of a multidisciplinary team (MDT) with good guidance from bodies such as NICE.

\section{From: Michael Jenkins}

Since we published the Editorial [1] regarding the proposed NICE (UK National Institute for Health and Care Excellence) guidelines on the management of abdominal aortic aneurysm (AAA), the final guidelines have now been published [2]. Following a massive response during the consultation period, NICE has listened to stakeholders and considered the implications of limited patient and clinician choice in the initial proposal. The final document reflects the concerns raised. Crucially, this gives autonomy to the individual doctor-patient relationship and recognizes that there may be some clinical situations when EVAR will be the optimum choice for patients under certain circumstances. We do not underestimate the scope of this change and the potential internal political difficulties involved in coming to this decision.

Although, where appropriate, based on fitness and likely life expectancy, open surgery remains the treatment of choice, endovascular aortic repair (EVAR) is now accepted as an option for some. This allows sensible discussion in a multidisciplinary team (MDT) setting as to the best treatment strategy for an individual patient. In our opinion, the question of anatomy is very important here. Restricting standard EVAR to within the IFU of the device being used should maximize the durability potential. For cases outside the conventional IFU of most devices, the situation is more difficult. Younger and fitter patients should be considered for open surgery, but there should not be a 'knee-jerk' move to custom devices without careful consideration of life expectancy and potential patient benefit. Prospective monitoring of the short- and long-term outcomes of such devices is vital, and we endorse the recommendation by NICE in this respect.

Importantly, these recommendations strike a pragmatic balance between the most durable treatment of open surgery for those fit enough to benefit from it, but at the same time, giving an opportunity for endovascular intervention (where appropriate) for those not suitable for open surgery. The revised recommendations empower patients and clinicians to consider the pros and cons of aneurysm treatment and should ensure sufficient focus is given to the decision-making process to best address the needs of the individual patient. It will be interesting to see if there is any further shift in the ratio between EVAR and open surgery over the next few years.

The ability to continue with both standard and custom EVAR is also crucial in furthering research and development into better endovascular devices for the future. Despite the current need for a partial return to open surgery, we must continue to strive for a more minimally invasive, patient-friendly and durable endovascular solution for the patients of tomorrow.

\section{Compliance with Ethical Standards}

Conflict of interest The authors declare that they have no conflict of interest. 


\section{References}

1. Uberoi R, Jenkins M. Is this the end for EVAR? Cardiovasc Intervent Radiol. 2020;43:169-71. https://doi.org/10.1007/s00270-019-02361-z.

2. Abdominal aortic aneurysm: diagnosis and management NICE guideline [NG156] Published date: 19 March 2020. https://www.nice.org.uk/ guidance/ng 156/chapter/Finding-more-information-and-resources.

3. NICE GDC committee statement; bit.ly/NICEAAA GDCstatement.

Publisher's Note Springer Nature remains neutral with regard to jurisdictional claims in published maps and institutional affiliations. 